\title{
That by which it is what it is
}

On page 356, John Gillies and colleagues describe a learning journey undertaken by RCGP Scotland in an attempt to define the essence of general practice. ${ }^{1}$ The context is the hostile intellectual environment perhaps best illustrated by Tony Delamothe's distressingly narrow and politically gullible article, published in the $B M J$ in November of last year. ${ }^{2}$ Clearly captivated by his own clever title, A good QOFfing whine, Delamothe, in his role as the Journal's Deputy Editor, is intent on dismissing the immeasurable and the ineffable from the discourse and analysis of medical care: 'In the current financial and political climate is it wise to defend primary care solely by invoking its warm fuzzy heart, beating away in its black box, far from the close scrutiny of all but its adepts?'

The problem is that the warm fuzzy heart is the essence - that by which it is what it is. Delamothe implies that there have been no attempts to explain the black box of general practice other than to an inner circle but this is simply not true. The paper by Gillies and colleagues is the latest of many worthwhile attempts to open up the black box to scrutiny. The key is that a thorough grasp of the science of medicine is essential but insufficient for the care of patients in general practice. All attempts to explain the more that is needed must inevitably draw on insights from beyond medical science and, perhaps because of this, they have not been granted serious attention by Tony Delamothe and those many others who take pride in their capacity for hard, if destructive, thinking.

GPs, in their daily meetings with patients confront undifferentiated, undiagnosed, and often undiagnosable, illness and suffering. They work at the point where the territory of human suffering meets the map of medical science and there is always a gap between the map and the territory. ${ }^{3}$ The gap contains the as yet immeasurable, the not yet knowable, alongside the perhaps forever unknowable and the ineffable. The possibility for new knowledge and for creative thinking about old knowledge exists in the gap and only through exploring the gap can we hope to improve the map. As Samuel Taylor Coleridge wrote in his Notebooks: 'Our ignorance, with all the intermediates of obscurity, is the condition of our everincreasing knowledge.'4 All the challenge, creativity, and potential innovation are located in the gap of ignorance, which exists between the map and the territory.

A convincing explanation of the persistence of the ineffable in the face of the enormous advances of scientific analysis comes from George Steiner and his description of music. ${ }^{5} \mathrm{He}$ writes: 'The more captive our delight, the more insistent our need of and "answering to" a piece of music, the more inaccessible are the reasons why. ... Music authorises, invites the conclusion that the theoretical and practical sciences, that rational investigation will never map experience exhaustively. That there are phenomena "at the centre" ... which will endure, boundlessly alive and indispensable, but "outside". This is, quite straightforwardly, the proof of the meta-physical. Music is significant to the utmost degree; it is also, strictly considered, meaningless. There abides its "transgression" beyond intellect.' All arenas of human endeavour touch on the not yet known and the unknowable. Medicine too must find a way of accommodating the ineffable.

GPs, alongside Italo Calvino's evocation of the god Mercury, ${ }^{6}$ find themselves 'between universal laws and individual destinies, between the forces of nature and the forms of culture, between the objects of the world and all thinking subjects'. According to Calvino, the necessary mercurial attributes are to be, 'light and airborne, astute, agile, adaptable, free and easy'. The attributes discovered on the learning journey described by Gillies and his colleagues are not so dissimilar: a robust intellect and a passion for knowledge, altruism and commitment, awareness of justice, integrity, respect for patients, empathy and emotional awareness, and the capacity for innovation and for working with others. Could there be a more inspiring list for young people considering a future profession?

The findings of the learning journey suggest that young doctors in training for general practice recognise the importance of tradition and values in instilling and maintaining professional motivation across generations and there is a clear request from young doctors for more explicit teaching and fostering of these. The task of making the medical map useful to those trapped within the territory of suffering is fraught with uncertainty because of the vast extent and infinite variation of the territory and because of the still rudimentary nature of the map. The task demands wisdom and judgment as much as knowledge and these are difficult to teach - but by no means impossible.

The foundation of wisdom is doubt. In Karl Weick's definition, 'wisdom is an attitude taken by persons toward the beliefs, values, knowledge, information, abilities, and skills that are held, a tendency to doubt that these are necessarily true or valid and to doubt that they are an exhaustive set of those things that could be known." Protocols derived from population studies cannot be identically applied to very different individuals. The capacity to doubt the universal relevance of such rules enables the wisdom to apply them flexibly and to improvise effectively in unfamiliar situations. Professionals without such doubt and wisdom finds themselves paralysed in situations for which there are no clear-cut rules and where the protocols do not appear to fit.

Contemporary rhetoric emphasises the imperative of patient-centred care and yet the core of health care is not a lonely patient but a series of unique human relationships between an individual patient and an individual healthcare professional. The longest lasting of these relationships usually exists between the patient and his or her GP because, alone of all healthcare professionals working in the NHS, the GP is not required to discharge the patient. Patients, consulted by RCGP Scotland during the learning journey, emphasised the importance of these continuing relationships and the explicit value, consistently attached to them by both doctors and patients, irritates and inconveniences those prioritising bureaucratic, organisational, and technical solutions in health care. Yet, when trying to apply the map of medicine to the territory of suffering, knowing the patient remains at least as important as knowing the disease, ${ }^{8}$ because there are always two sides of the jigsaw to be fitted together. Relationships are unpredictable because both parties are 
changed by them and they are so multifaceted that it is very difficult to measure them in any meaningful sense. Yet these ineffable, immeasurable, uncertain relationships between doctors and patients remain very close to the warm fuzzy heart of general practice and much of its essence.

\section{Iona Heath,}

GP, Caversham Group Practice and

Chair of the RCGP International Committee. essence of general practice: a learning journey in progress. Br I Gen Pract 2009; 59: 356-363.

2. Delamothe T. A good QOFfing whine. BMJ 2008; 337: a2632.

3. Korzybski A. Science and sanity: an introduction to nonAristotelian systems and general semantics. Lancaster, PA: The International Non-Aristotelian Library, 1933.

4. Coleridge ST. Notebooks, Volume 3, 1809-1818. In Holmes R. Coleridge: darker reflections, 1804-1834. London: Harper Collins, 1998.

5. Steiner G. Errata: an examined life. Blaine, WA: Phoenix, 1998.

6. Calvino I. Six memos for the millennium: Charles Eliot Norton Lectures, 1985. London: Vintage, 1996.

7. Weick, KE. The collapse of sensemaking in organizations: The Mann Gulch disaster. Administrative Science Quarterly 1993; 38: 628-652.
8. McCormick J. Death of the personal doctor. Lancet 1996; 348: $667-668$.

DOI: 10.3399/bjgp09X420545

ADDRESS FOR CORRESPONDENCE

Iona Heath

Caversham Group Practice, 4 Peckwater

Street, London NW5 $2 U$.

E-mail: iona.heath@dsl.pipex.com

\section{Getting a grip on guidelines: how to make them more relevant for practice}

Evidence-based guideline development can be seen as one of the major achievements in efforts to improve patient care in the last decade. Well developed clinical guidelines provide professionals, patients, and policy makers with information on how to manage health problems appropriately within day-today practice. The development of guidelines has made an enormous progress in the last 10-15 years with many guideline development programmes, such as those of NICE, SIGN, and medical colleges, using established methods and procedures, according to AGREE Collaboration criteria. ${ }^{1}$

New developments related to searching and grading evidence (SEARCH, GRADE), and adapting guidelines to local context (ADAPTE) aim at making guideline development better and more effective. Guidelines have had a major impact on care in general practice in some countries; for instance, in the Netherlands most problems seen in general practice are covered by national evidence-based guidelines developed by the scientific body with now around $75 \%$ of the decisions in line with these guidelines. ${ }^{2}$

An exciting next step in this development is to link evidence-based guidelines to incentives for performance, as initiated in the Quality and Outcomes Framework (QOF). A worldwide collaboration between guideline developers has been created with over 70 organisations as members (Guidelines International Network). A new world of clinicians, epidemiologists, and professional guideline developers with their own meetings, procedures, and vested interests has grown. This highlights also one of the risks of guideline development, that is, becoming increasingly 'institutionalised', top-down driven by research findings, notably randomised controlled trials, imposed upon practice. A balanced view of risks and benefits of clinical guidelines is therefore needed, in which preferences of patients and demands of practice and policy, are matched with the achievements of science.

Against this background, a number of persistent problems can be observed in guideline development that come forward in the paper comparing depression guidelines from seven different countries, in this issue of the $B J G P{ }^{3}$ One of the problems identified is that many clinical guidelines still do not yet meet internationally accepted quality criteria, as defined in the AGREE instrument. ${ }^{1}$ For instance, the rigour of development scores for the seven studied depression guidelines ranged from 1-64\%. ${ }^{3}$ In addition, experiences with guideline development indicate that there is a risk of substantial bias in guidelines. ${ }^{3}$ Appropriate evidence is lacking for many recommendations and, even when evidence is available, the final recommendations for practice are often largely a reflection of local culture or personal views of the guideline developers. Guideline developers in different countries often come up with different advice for decision making in practice, partly because they refer to different, not overlapping sources of evidence. ${ }^{4,5}$ Normative and cultural opinions about the value of specific performance often play an important role in defining recommendations for practice without making these explicit. ${ }^{6,7}$

Many recommendations in clinical guidelines aim at 'ideal patients', usually adult patients without any comorbidity, and are not very well tailored to actual patient care and real patients. In normal general practice, many patients, particularly patients with chronic multi-morbidity, have a combination of (interacting) problems, which make appropriate performance and decision making more complex. ${ }^{8}$ There is an obvious development towards integrated care with increasing collaboration between disciplines, while most clinical guidelines do not yet focus on this complexity. A recent publication relating to GPs' management of depression has cast doubts on whether essential aspects of day-to-day care, notably comorbidity, have been considered in evidence-based guidelines and QOF indicators. ${ }^{9,10}$ Many clinical guidelines are still mono-disciplinary, often written by specialists from tertiary care, who have a different type of patient in mind.

Guideline development is time consuming and expensive, about €100 000-200 000 per guideline, and the question is, if this is costeffective. It may be, if guidelines were valid and had a wide impact on health care. 